\title{
The complex relationship between pediatric cardiac surgical case volumes and mortality rates in a national clinical database
}

\author{
Karl F. Welke, MD, ${ }^{\mathrm{a}}$ Sean M. O’Brien, PhD, ${ }^{\mathrm{b}}$ Eric D. Peterson, MD, MPH, ${ }^{\mathrm{b}}$ Ross M. Ungerleider, MD, MBA, \\ Marshall L. Jacobs, MD, ${ }^{\text {d }}$ and Jeffery P. Jacobs, MD ${ }^{\mathrm{e}}$
}

Objective: We sought to determine the association between pediatric cardiac surgical volume and mortality using sophisticated case-mix adjustment and a national clinical database.

\begin{abstract}
Methods: Patients 18 years of age or less who had a cardiac operation between 2002 and 2006 were identified in the Society of Thoracic Surgeons Congenital Heart Surgery Database (32,413 patients from 48 programs). Programs were grouped by yearly pediatric cardiac surgical volume (small, <150; medium, 150-249; large, 250-349; and very large, $\geq 350$ cases per year). Logistic regression was used to adjust mortality rates for volume, surgical case mix (Aristotle Basic Complexity and Risk Adjustment for Congenital Heart Surgery, Version 1 categories), patient risk factors, and year of operation.

Results: With adjustment for patient-level risk factors and surgical case mix, there was an inverse relationship between overall surgical volume as a continuous variable and mortality $(P=.002)$. When the data were displayed graphically, there appeared to be an inflection point between 200 and 300 cases per year. When volume was analyzed as a categorical variable, the relationship was most apparent for difficult operations (Aristotle technical difficulty component score, $>3.0)$, for which mortality decreased from $14.8 \%(60 / 406)$ at small programs to $8.4 \%(157 / 1858)$ at very large programs $(P=.02)$. The same was true for the subgroup of patients who underwent Norwood procedures (36.5\% [23/63] vs 16.9\% [81/479], $P<.0001)$. After risk adjustment, all groups performed similarly for low-difficulty operations. Conversely, for difficult procedures, small programs performed significantly worse. For Norwood procedures, very large programs outperformed all other groups.
\end{abstract}

Conclusion: There was an inverse association between pediatric cardiac surgical volume and mortality that became increasingly important as case complexity increased. Although volume was not associated with mortality for low-complexity cases, lower-volume programs underperformed larger programs as case complexity increased.

Earn CME credits at

http://cme.ctsnetjournals.org

The drive to quantify and publicly report hospital quality has intensified the search for comparable quality measures. Because of its simplicity and ready availability, surgical volume is one of the most often cited metrics. The relationship between hospital surgical volume and in-hospital mortality for coronary artery bypass grafting surgery has been one of the

\footnotetext{
From the Division of Cardiothoracic Surgery, ${ }^{\text {a }}$ Oregon Health and Science University, Portland, Ore; the Outcomes Research and Assessment Group, ${ }^{\mathrm{b}}$ Duke Clinical Research Institute, Durham, NC; Division of Pediatric Cardiothoracic Surgery, Department of Surgery, Case Western Reserve University, ${ }^{\mathrm{c}}$ Cleveland, Ohio; Drexel University College of Medicine, ${ }^{\mathrm{d}}$ Philadelphia, $\mathrm{Pa}$; the Congenital Heart Institute of Florida, ${ }^{\mathrm{e}}$ Saint Petersburg and Tampa, Fla.

Read at the Eighty-eighth Annual Meeting of The American Association for Thoracic Surgery, San Diego, Calif, May 10-14, 2008.

Received for publication May 9, 2008; revisions received Nov 19, 2008; accepted for publication Dec 19, 2008.

Address for reprints: Karl F. Welke, MD, Division of Cardiothoracic Surgery L353, Oregon Health and Science University, 3181 S.W. Sam Jackson Park Road, Portland, OR 97239-3098 (E-mail: welkek@ohsu.edu).

J Thorac Cardiovasc Surg 2009;137:1133-40

$0022-5223 / \$ 36.00$

Copyright (C) 2009 by The American Association for Thoracic Surgery

doi:10.1016/j.jtcvs.2008.12.012
}

most studied. Importantly, the strength of the relationship has depended on the database used. The inverse association between hospital volume and mortality is more apparent in administrative data than in clinical data. ${ }^{1,2}$ This is in part due to the more sophisticated risk adjustment possible with clinical data and differences in the cohorts of hospitals. ${ }^{3}$

Although less studied, the relationship between hospitals' pediatric cardiac surgical volumes and mortality rates has been the subject of several previous investigations. ${ }^{4-9}$ These studies have used either administrative data or single-state clinical data. The largest and most recent of these studies demonstrated an inverse and nonlinear association between volume and mortality after adjustment for patient age and surgical case mix. ${ }^{9}$ Although this study benefited from a national database, the reliance on administrative data limited the ability to adjust for patient-level risk factors. Surgical case-mix designation was also limited by administrative coding.

The purpose of our study was to determine the relationship between program surgical volume and mortality after pediatric cardiac surgery. For the investigation, we used clinical data from the Society of Thoracic Surgeons (STS) Congenital Heart Surgery Database. We first examined the raw association between overall surgical volume and mortality. We then investigated how this association is affected by adjustment for both patient-level risk factors and surgical case mix. 


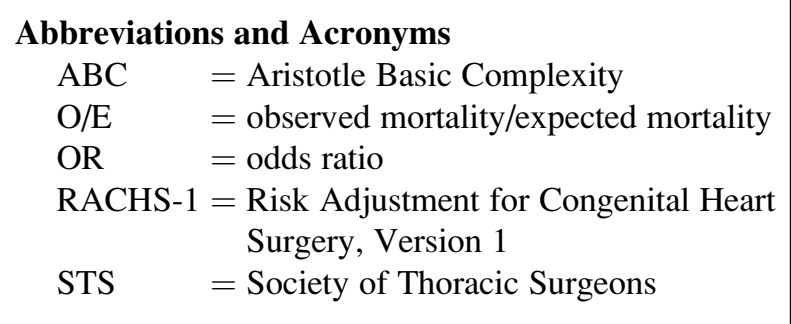

\section{MATERIALS AND METHODS Database}

This study was designed as a retrospective cohort analysis. We obtained data from the STS Congenital Heart Surgery Database. ${ }^{10}$ The STS maintains the provider-led voluntary cardiac surgical clinical database as a means of supporting national quality-improvement efforts. The database grew from 6790 operations from 20 participants during calendar year 2002 to 19,853 operations from 56 participants during calendar year 2006. Database participants are either hospitals or surgical groups that are affiliated with hospitals at which cardiac surgery is performed. Each record corresponds to a primary cardiac surgical procedure. Data elements include basic patient demographic information, comorbidities and preoperative risk factors, diagnoses, type of operation, and outcomes, including in-hospital mortality, 30-day mortality, major morbidity, and postoperative length of stay.

\section{Study Population}

The study population consisted of patients age 18 years or less who underwent a cardiovascular operation at an STS-participating program between January 1, 2002, and December 31, 2006. Programs were excluded if they had more than $10 \%$ missing data on key study variables, including preoperative risk factors, noncardiac abnormalities, discharge mortality, and length of stay. Ten programs were deleted because of data quality issues, leaving 48 programs in the study cohort. Seven of the 48 programs performed operations at 2 hospitals. In each case the vast majority of operations were performed at one of the hospitals. Patients were initially included if they underwent one of the cardiovascular procedures for which the Aristotle Basic Complexity (ABC) Score and/or the Risk Adjustment for Congenital Heart Surgery, Version 1 (RACHS-1) complexity level is defined. ${ }^{11,12}$ In addition to cardiovascular procedures, the $\mathrm{ABC}$ score is also defined for 13 noncardiovascular procedures that were excluded from the analysis. Patients undergoing one of these 13 noncardiovascular procedures were only included if the noncardiovascular procedure was performed concomitantly with a cardiovascular procedure and was not the primary procedure of the operation. In addition, patients weighing less than or equal to $2500 \mathrm{~g}$ undergoing patent ductus arteriosus ligation as their primary procedure were excluded from the analysis. In cases in which patients had multiple operations during the same hospital admission, only the first operation was analyzed. Finally, patients were excluded if they were missing data on 2 key risk-adjustment variables: age and weight.

\section{Definition of Surgical Volumes}

Average annual program volumes were based on operations submitted to the STS database between January 1, 2002, and December 31, 2006. Case volumes were defined by counting the number of admissions for which the index operation (first operation of the hospitalization) was cardiovascular. The average annual program volume was calculated by dividing the number of admissions during the study period by the number of months that the program participated in the database during the study period and then multiplying by 12 . For tabular presentation, volumes were categorized into 4 groups: less than 150,150 to 249,250 to 349 , and 350 or more cases per year. These categories were chosen to ensure an adequate sample size (num- ber of events) in each category when analyzed in the overall population, as well as subgroups. The association between volume and mortality was also explored without categorizing volume, as described below.

We repeated the calculation of surgical volumes by counting the total number of cardiovascular operations (instead of the number of admissions). When these operation-based volumes were compared with the original admission-based volumes, the Pearson correlation was 0.99 . Hence there is little risk that a program's volume status was misclassified based on the decision to define volume in terms of admissions or operations.

\section{End Point}

Our primary end point was in-hospital mortality, which was defined as death during the same hospitalization as the operation, regardless of timing.

\section{Analysis of Mortality}

Unadjusted mortality rates were compared across volume categories by using logistic regression with generalized estimating equations to account for correlation of outcomes within hospitals. A linear trend test was conducted by assigning integer scores to the volume categories and testing whether the coefficient for these integer scores was zero. Next, a generalized estimating equation logistic regression model was used to assess the association between volume and mortality while adjusting for patient-level risk factors and surgical case mix.

Patient-level risk factors. The following variables were included in the adjusted mortality model: age (modeled as a piecewise linear variable with knots at 30 days and 1 year); age-for-weight-and-sex $z$ score; interaction between age and age-for-weight-and-sex $z$ score; preoperative stay for more than 2 days; number of prior operations $(0,1$, and $\geq 2)$; renal failure or dialysis; acidosis, circulatory support, or shock; preoperative ventilatory support or tracheostomy; asplenia, polysplenia, or a22q11 deletion; DiGeorge syndrome; Down syndrome; procedure or procedure group; and operation date (modeled as a linear trend).

Modeling of volume. Volume was initially modeled as a categorical variable, with categories corresponding to less than 150,150 to 249,250 to 349 , and 350 or more cases per year. Because categorization can reduce statistical power, the analysis was also performed by entering volume in the model as a single continuous linear variable. This approach was used for testing the overall null hypothesis of no association between volume and mortality. Finally, to explore possible nonlinear volume effects, volume was analyzed by using restricted cubic splines. Knots for the spline function were placed at 150, 250, and 350 cases per year. Because the shape of a spline function might depend on the choice of arbitrary knot locations, the model was subsequently repeated by using 2 alternative specifications: knots at 100, 300, and 500 cases and knots at 200, 300, and 450 cases.

Adjustment for type of procedure. Because of the large number of procedures with small sample sizes, it was not feasible to adjust for procedure identity by including a separate term for each individual procedure. Instead, a coarser covariate adjustment was performed by grouping procedures of similar complexity. Any procedure with at least 20 deaths recorded in the database was considered to be its own stratum. All remaining procedures were stratified by ABC level and RACHS-1 category. The possible values of the ABC level were 1, 2, 3, 4, and "unassigned." The possible values of the RACHS-1 level were 1, 2, 3, 4, 6, and "unassigned." "Unassigned" indicates that the procedure type was not included by the developers of the ABC or RACHS-1 methodology. To perform the covariate adjustment, we grouped procedures according to the 30 unique combinations of $\mathrm{ABC}$ and RACHS-1 (5 levels of ABC [including "unassigned'] $\times 6$ levels of RACHS1 [including "unassigned"'] $=30$ unique combinations). Strata with zero deaths do not provide information for studying the volume-outcome association and were therefore excluded. The remaining 28 combinations were entered into the logistic regression model as a set of category indicator variables. Subgroup analyses. In addition to studying the overall volume-outcome association, we also assessed the volume-outcome association 
separately for low-difficulty and high-difficulty procedures. We did this by including an interaction between volume and difficulty in the regression models. Procedures were classified by difficulty based on the difficulty component of the Aristotle score. The categories were Aristotle difficulty of 3.0 or less versus 3.0 or greater. Procedures that were not classified by using the Aristotle system were excluded from this analysis. We also performed a separate subgroup analysis to assess the volume-outcome association specifically for the Norwood operation. The Norwood operation was the only procedure with enough events to make a standalone analysis informative.

Sensitivity analysis. To determine whether the observed volumeoutcome association was an artifact of unusually high performance at just 1 or 2 high-volume hospitals, we repeated the analysis 3 ways: (1) after excluding the single participant with the largest average annualized number of index operations, (2) after excluding the 2 participants with the largest average annualized number of index operations, and (3) after excluding the participant in the highest volume category with the lowest observed mortality to expected mortality $(\mathrm{O} / \mathrm{E})$ ratio. The first program participated for 3 years, contributed 2080 cases to the regression analysis, had an average annualized volume of 850 cases per year, and had the third lowest $\mathrm{O} / \mathrm{E}$ ratio among participants in the highest volume category. The second program participated for 5 years, contributed 3279 cases to the regression analysis, had an average annualized volume of 683 cases per year, and had the second lowest $\mathrm{O} / \mathrm{E}$ ratio among participants in the highest volume count. This program contributed more cases to the regression analysis than any other participant. (Note: the average annualized volume does not correspond to the number of cases in the regression analysis because of differing inclusion/exclusion criteria for calculating participant volumes and analyzing outcomes.) The third program participated for 5 years, contributed 2769 cases to the analysis, had an average annualized volume of 600.6 , and had the lowest $\mathrm{O} / \mathrm{E}$ ratio among participants in the analysis.

If the observed volume-outcome association disappeared after excluding these hospitals, this might indicate that the apparent volume-outcome association was an artifact of unusual results at just 1 or 2 high-volume hospitals.

\section{RESULTS}

We identified 32,413 operations in the STS Congenital Heart Surgery Database from 2002 to 2006 that met our inclusion criteria. These operations took place at 48 programs. The average annual pediatric cardiac surgical volume at these programs ranged from 66 to 850 cases (Figure 1).

Patient-level risk factors and surgical case mix varied by volume category (Table 1). The case mix at larger programs included a higher percentage of younger patients. As a result, operations done at larger-volume programs more frequently involved lower-weight patients. The case mix at larger-volume programs also included a greater percentage of more complex operations. Patient demographics and preoperative risk factors did not differ by volume category.

The overall unadjusted mortality rate for the cohort was $3.7 \%$. When programs were grouped into 4 volume categories, the overall unadjusted mortality rate at small programs was lower than that at medium-volume programs $(4.0 \%$ vs $4.1 \%)$ and slightly higher than those at large- and very large-volume programs $(3.8 \%$ and $3.3 \%$, respectively; Table 2). When mortality risk was modeled as a function of program volume categories, the $\mathrm{c}$ statistic was low (0.53), indicating that volume alone was a poor predictor of mortality. Adjustment for patient risk factors and surgical case mix improved the discrimination of the model

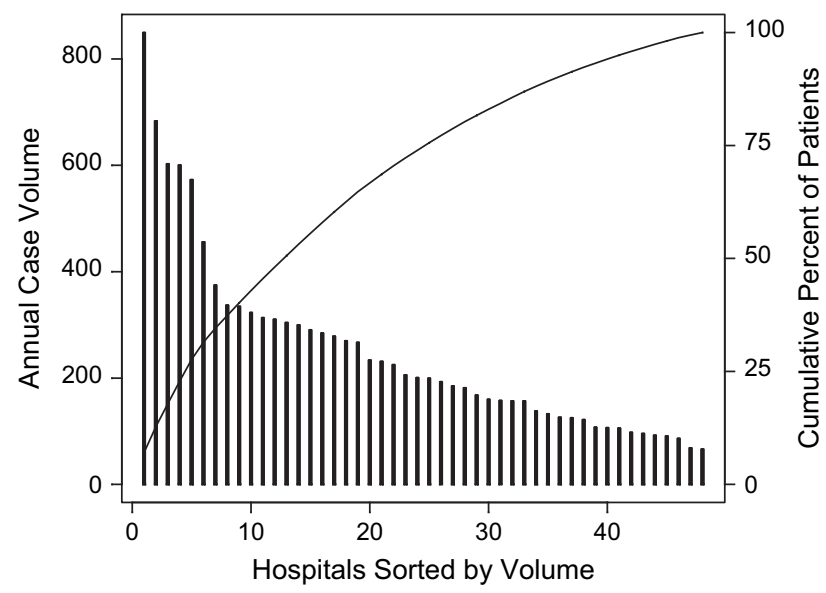

FIGURE 1. Distribution of hospital average annual case volumes.

substantially (c statistic $=0.84)$. After adjustment, the mortality rates at medium and large programs were similar to the mortality rates at very large programs (odds ratios [OR], 1.05 and 1.14, respectively), but compared with very large programs, mortality at small programs was significantly higher (OR, 1.51; $P=.0005$; Table 3$)$.

We then subdivided the case mix by difficulty to see whether there was a differential effect of volume (Table 3 ). For low-difficulty operations (defined as Aristotle difficulty $\leq 3.0)$, all 4 volume groups performed similarly $(P=.29$, test of trend). However, for high-difficulty operations (defined as Aristotle difficulty $>3.0$ ), small programs had substantially higher adjusted mortality relative to very high-volume programs $(\mathrm{OR}, 2.41 ; P<.0001)$. Mortality rates for high-difficulty operations performed at medium and large programs were similar to the mortality rates at very large programs. We then examined the volume-mortality relationship for the Norwood procedure. This operation was chosen because of the high level of system knowledge and coordination needed to achieve success. For the Norwood procedure, very highvolume programs outperformed all other volume groups.

To further investigate the volume-mortality relationship, we analyzed volume as a continuous variable and used logistic regression to adjust for patient-level risk factors and surgical case mix. We found an inverse relationship between overall surgical volume as a continuous variable and mortality $(P=.002)$. We then plotted the ORs for risk-adjusted mortality by using a volume of 800 cases per year as the arbitrary reference (OR, 1.0). For overall surgical volume, the slope of the volume-mortality curve was steepest below an infection point, which occurred between 200 and 300 cases per year (Figure 2). This nonlinear effect was not significant for low-complexity cases $(P=.06$, test of no volume-mortality association; Figure 3) but was consistent for high-complexity cases $(P=.007$, test of no volume-mortality association; Figure 3$)$ and the Norwood operation $(P<.001$, test of no volume-mortality association; Figure 4). Importantly, the reliability of the inflection point cannot be assured because 


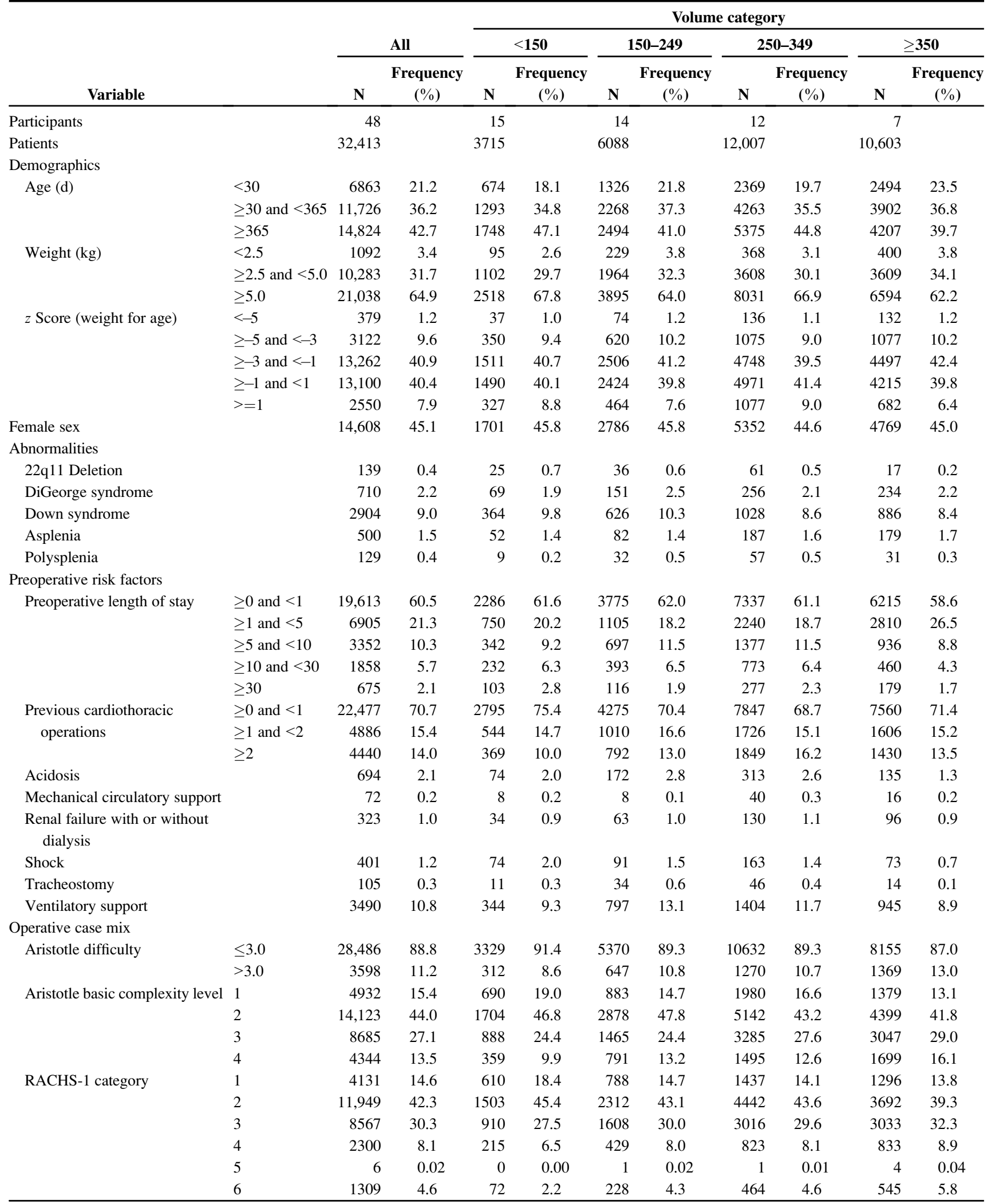


TABLE 2. Raw mortality rates (percentage mortality and $95 \%$ confidence interval) by volume category

\begin{tabular}{lcccccc}
\hline & \multicolumn{5}{c}{ Volume category } \\
\cline { 2 - 7 } Procedure subgroup & All & $<\mathbf{1 5 0}$ & $\mathbf{1 5 0 - 2 4 9}$ & $\mathbf{2 5 0 - 3 4 9}$ & $\geq \mathbf{3 5 0}$ & $\boldsymbol{P}$ value* \\
\hline All procedures & $3.7(3.3-4.1)$ & $4.0(2.9-5.0)$ & $4.1(3.1-5.2)$ & $3.8(3.3-4.2)$ & $3.3(2.7-3.8)$ \\
Aristotle difficulty $\leq 3.0$ & $2.6(2.3-2.9)$ & $2.7(1.9-3.4)$ & $2.9(2.2-3.6)$ & $2.9(2.5-3.2)$ & $2.2(1.9-2.5)$ & .101 \\
Aristotle difficulty $>3.0$ & $9.8(8.4-11.1)$ & $14.8(11.4-18.2)$ & $11.1(7.7-14.5)$ & $9.3(7.6-11.0)$ & $8.4(6.2-10.7)$ & .020 \\
Norwood procedures & $21.3(18.4-24.3)$ & $36.5(27.4-45.7)$ & $24.2(17.7-30.7)$ & $22.7(18.9-26.6)$ & $16.9(14.0-19.9)$ & $<.0001$ \\
\hline
\end{tabular}

$* P$ value for linear trend across volume groups.

as a result of the number of programs in the cohort, the $95 \%$ confidence intervals were not sufficiently narrow. When the analysis was repeated by using 2 alternative specifications for the knot locations, the shape of the curves was similar, but the location of the inflection point increased to between 300 and 400 cases per year.

To further assess the stability of our results, we conducted a series of sensitivity analyses. After removal of the largest and 2 largest programs from the cohort, there was no substantial difference in our findings. Similarly, removal of the lowest-mortality program did not appreciably alter the results.

\section{DISCUSSION}

After risk adjustment for patient-level variables and surgical case mix, there was an inverse relationship between over-

TABLE 3. Association between annual case volume and mortality*

\begin{tabular}{|c|c|c|c|c|}
\hline Volume category & $\mathbf{N}$ & Deaths & $\begin{array}{c}\text { Adjusted odds } \\
\text { ratio } \\
(95 \% \text { confidence } \\
\text { interval) } \\
\end{array}$ & $P$ value \\
\hline \multicolumn{5}{|l|}{ All procedures } \\
\hline$\geq 350$ & 10,570 & 346 & Reference & $.004 \dagger$ \\
\hline $250-349$ & 11,978 & 450 & $1.05(0.86-1.29)$ & .63 \\
\hline $150-249$ & 6051 & 250 & $1.14(0.84-1.55)$ & .41 \\
\hline$<150$ & 3681 & 148 & $1.51(1.19-1.90)$ & .0005 \\
\hline \multicolumn{5}{|l|}{$\begin{array}{l}\text { Low-difficulty } \\
\text { procedures }\end{array}$} \\
\hline$\geq 350$ & 8663 & 188 & Reference & $.29 \dagger$ \\
\hline $250-349$ & 10,252 & 295 & $1.16(0.87-1.53)$ & .31 \\
\hline $150-249$ & 5104 & 148 & $1.08(0.76-1.52)$ & .68 \\
\hline$<150$ & 3229 & 86 & $1.21(0.87-1.69)$ & .26 \\
\hline \multicolumn{5}{|l|}{$\begin{array}{l}\text { High-difficulty } \\
\text { procedures }\end{array}$} \\
\hline$\geq 350$ & 1855 & 135 & Reference & $.0008 \dagger$ \\
\hline $250-349$ & 1636 & 138 & $0.89(0.69-1.15)$ & .38 \\
\hline $150-249$ & 894 & 79 & $1.22(0.81-1.84)$ & .35 \\
\hline$<150$ & 406 & 54 & $2.41(1.89-3.06)$ & $<.0001$ \\
\hline \multicolumn{5}{|l|}{ Norwood procedures } \\
\hline$\geq 350$ & 479 & 81 & Reference & $<.0001 \dagger$ \\
\hline $250-349$ & 418 & 95 & $1.43(1.06-1.95)$ & .020 \\
\hline $150-249$ & 194 & 47 & $1.59(1.09-2.32)$ & .016 \\
\hline$<150$ & 63 & 23 & $2.91(1.98-4.28)$ & $<.0001$ \\
\hline
\end{tabular}

*The smaller numbers of operations in this table compared with those in Table 1 reflect the exclusion of patients with missing risk adjustment data. Although Table 1 includes the entire cohort, Table 3 excludes 89 patients who were in a procedure stratum with zero deaths (as mentioned in the methods section) and 44 patients with missing mortality status. $\dagger P$ for linear trend. all surgical volume as a continuous variable and mortality. When analyzed as a categorical variable, the relationship was most apparent for difficult operations. For one of the most complex procedures (the Norwood procedure), the largest programs had results that were significantly better than those of all other groups. Although volume alone was an unreliable discriminator of mortality, mortality rates adjusted for patient risk factors and surgical case mix suggest that in aggregate higher-volume programs achieve lower mortality rates for complex operations.

Although we did find a relationship between case volume and mortality, this finding must be interpreted with caution. The aggregation of programs into volume groups gave us sufficient statistical power to analyze important relationships but disguised individual programs. One should not conclude that all larger programs perform better than all smaller programs. Although on average this was true, there were low-volume programs that had low mortality rates and those that had volumes too low for any mortality rate difference to be observed. Importantly, our analysis highlights the unsuitability of volume alone, without adjustment for patient risk factors and surgical case mix, as a marker of quality. In general, a patient's own risk characteristics and level of disease burden account for the vast majority of his or her mortality risk, and the effect of program volume on the mortality risk of an individual patient is small.

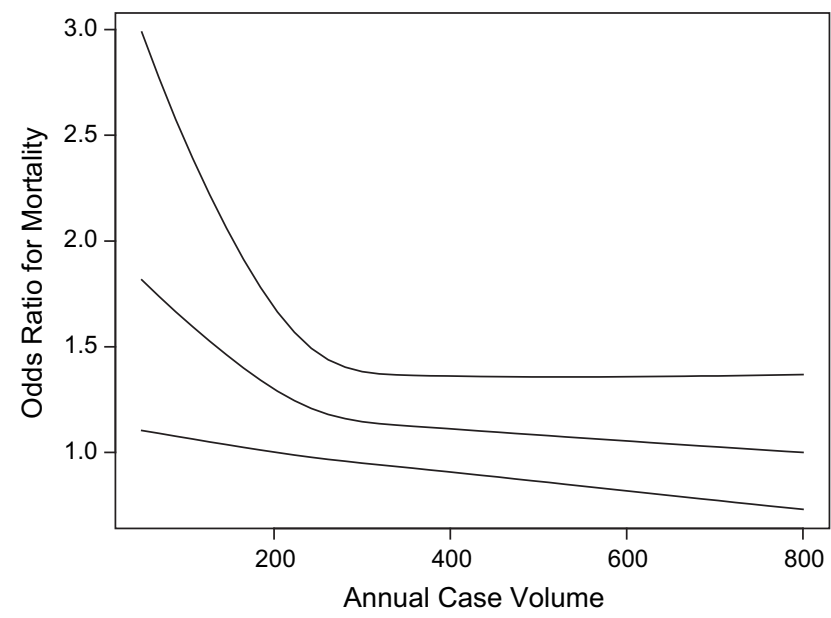

FIGURE 2. Association between overall annual volume and risk-adjusted mortality $(P=.002$, test of no volume-mortality association). 

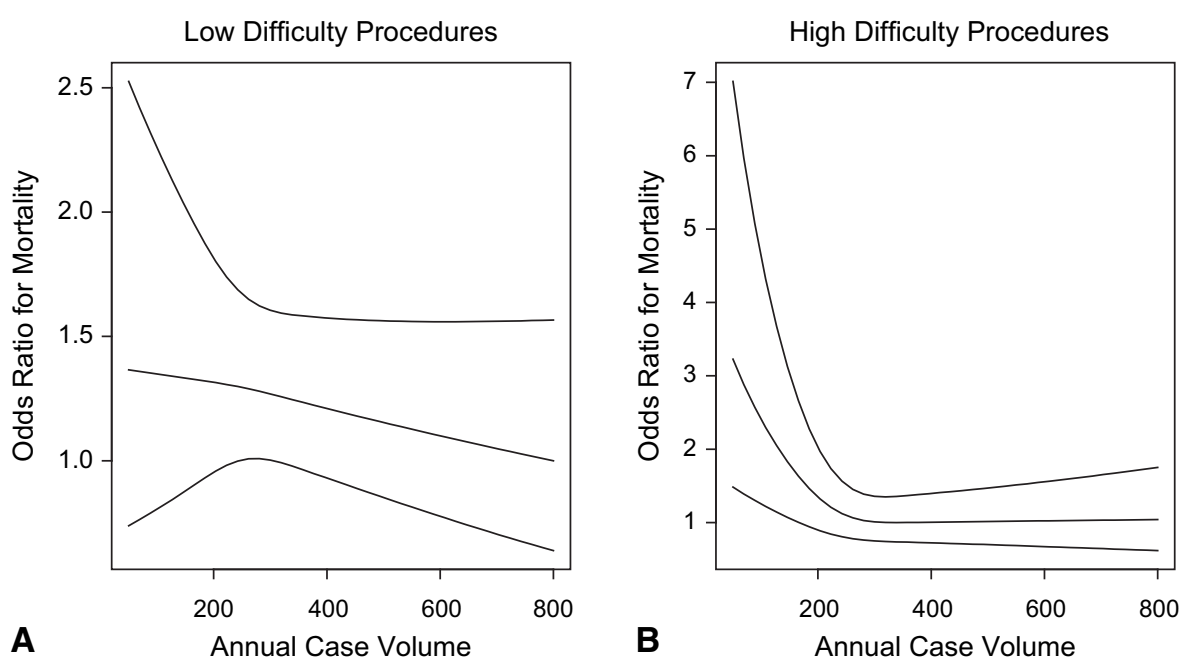

FIGURE 3. Association between surgical volume and risk-adjusted mortality by Aristotle difficulty (A, low difficulty, $\leq 3[P=.059$, test of no volumemortality association]; $\mathrm{B}$, high difficulty, $>3[P=.007$, test of no volume-mortality association $]$ ).

The results of this study complement those of the largest and most recent previous study of the volume-mortality relationship in pediatric cardiac surgery. ${ }^{9}$ Although the 2 investigations were done with different types of data, both found that larger programs performed better than smaller programs. The previous study used the Nationwide Inpatient Sample, which is an involuntary administrative database, which is a stratified cross-sectional sample that includes approximately $20 \%$ of all community (nonfederal) hospital discharges in the United States selected from a sampling frame that comprises approximately $90 \%$ of all hospital discharges in the United States. The STS Congenital Heart Surgery Database, as used in the present study, is a voluntary clinical database. As such, the participants are more likely to be higher-volume programs specializing in congenital

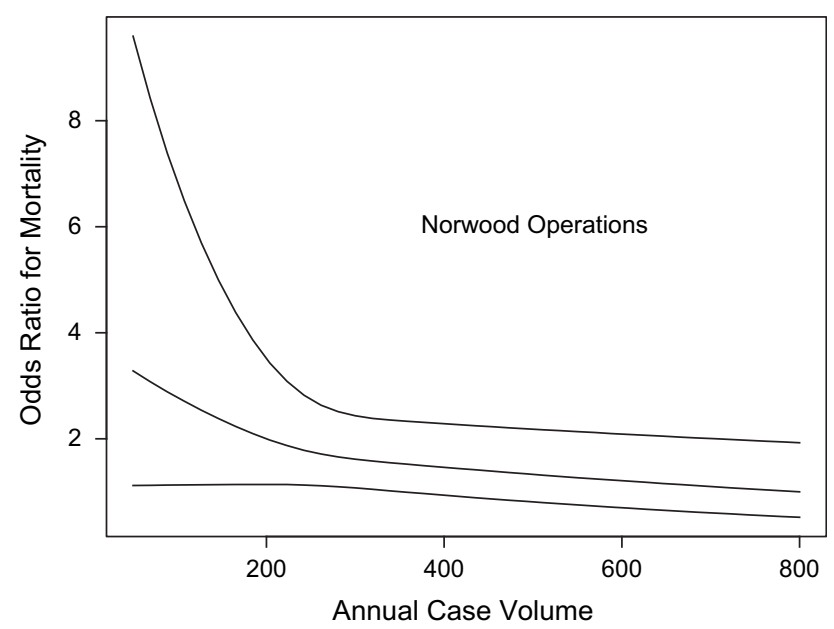

FIGURE 4. Association between hospital volume and risk-adjusted mortality for Norwood operations $(P<.001$, test of no volume-mortality association). cardiac surgery. Consequently, the volume categorizations differed between the 2 studies. Despite this population difference and the greater ability for risk adjustment with clinical data, the findings of the 2 studies were similar.

The limitations of this study are a result of the choice of the data source. The STS Congenital Heart Surgery Database was designed primarily for quality improvement. The database contains a self-selected group of centers that voluntarily chose to submit data. The data were collected by individuals at the institutions, many of whom have a stake in the outcomes of the program. Although some have questioned the validity of such data because of this involvement, the contributions of clinical personnel to the data collection process and the design of the database specifically for congenital cardiac surgery might result in higher-quality data and superior risk adjustment. Unfortunately, at the time of the data collection for this study, there was no data audit process to ensure accuracy. The consistency of the aggregate morality rates and the volume-mortality relationships with previous reports supports the quality of the database.

Because we did not have information on preoperative decision making, we were not able to assess the appropriateness of the operations performed. For example, for a certain diagnosis, a patient might receive an operation with a lower in-hospital mortality rate but lower long-term survival rather than a more appropriate operation with a higher up-front mortality and better long-term survival. In addition, the choice of the low-mortality operation might necessitate a second operation, resulting in a higher combined mortality rate. The lack of long-term follow-up limited our ability to compare the "true" outcomes important to congenital heart surgery, including not only mortality but also morbidity, functional status, and neurologic status. Because the overall mortality rate for our cohort was $3.7 \%$, these outcomes, rather than in-hospital mortality, are of importance to more than $96 \%$ of our 
patients. Future efforts need to investigate these outcomes. Notwithstanding these limitations, our study was conducted by using a national multicenter database with adequate power to generate current, stable mortality rates.

\section{CONCLUSION}

We found overall unadjusted volume to be a poor discriminator of mortality. However, after adjustment for patient risk factors and surgical case mix, larger programs achieved superior results for more complex operations. Many factors contribute to the mortality risk of a patient undergoing pediatric cardiac surgery. The relationship between volume and mortality is complex, making volume a difficult choice as a quality measure for pediatric cardiac surgery. Rather than accepting an imperfect proxy, the process measures and system characteristics for which volume is a surrogate need to be identified. The widespread implementation of these factors is likely to lead to substantial improvement in the outcomes of our operations.

\section{References}

1. Birkmeyer JD, Siewers AE, Findlayson EV, et al. Hospital volume and surgical volume in the United States. N Engl J Med. 2002;346:1128-37.

2. Peterson ED, Coombs LP, DeLong ER, Haan CK, Ferguson TB. Procedural volume as a marker of quality for CABG surgery. JAMA. 2004;291:195-201.

3. Welke KF, Peterson ED, Vaughn-Sarrazin MS, et al. Comparison of cardiac surgical volumes and mortality rates between the Society of Thoracic Surgeons and Medicare databases from 1993 through 2001. Ann Thorac Surg. 2007;84:1538-46.

4. Jenkins KJ, Newburger JW, Lock JE, et al. In-hospital mortality for surgical repair of congenital heart defects: preliminary observations of variation by hospital caseload. Pediatrics. 1995;95:323-30.

5. Hannan EL, Racz M, Kavey RE, Quagebeur JM, Williams R. Pediatric cardiac surgery: the effect of hospital and surgeon volume on in-hospital mortality. Pediatrics. 1998;101:963-9.

6. Sollano JA, Gelijns AC, Moskowitz AJ, et al. Volume-outcome relationships in cardiovascular operations: New York State, 1990-1995. J Thorac Cardiovasc Surg. 1999;117:419-28.

7. Chang RK, Klitzner TS. Can regionalization decrease the number of deaths for children who undergo cardiac surgery? A theoretical analysis. Pediatrics. 2002; 109:173-81.

8. Bazzani LG, Marcin JP. Case volume and mortality in pediatric cardiac surgery patients in California, 1998-2003. Circulation. 2007;115:2652-9.

9. Welke KF, Diggs BS, Karamlou T, Ungerleider RM. The relationship between hospital surgical case volumes and mortality rates in pediatric cardiac surgery: a national sample 1988-2005. Ann Thorac Surg. 2008;86:889-96.

10. The Society of Thoracic Surgeons Congenital Heart Surgery Database 2007 Congenital Report Executive Summary. Available at: http://www.sts.org/ documents/pdf/2007_Congenital_Report_Executive_Summary_-_All_Patients. pdf. Accessed May 8, 2008

11. Jenkins KJ, Gauvreau K, Newburger JW, Spray TL, Moller JH, Iezzoni LI. Consensus-based method for risk adjustment for surgery for congenital heart disease. J Thorac Cardiovasc Surg. 2002;123:110-8.

12. Lacour-Gayet F, Clark D, Jacobs J, et al. The Aristotle score: a complexityadjusted method to evaluate surgical results. Eur J Cardiothorac Surg. 2004; 25:911-24.

\section{Discussion}

Dr J. William Gaynor (Philadelphia, $\mathrm{Pa}$ ). I would like to congratulate Dr Welke and his coinvestigators for a very important and interesting study. They have used data from the STS congenital database to investigate the relationship between center-based surgi- cal volume and outcome as assessed based on hospital mortality. This is an important and controversial topic because center volume has been used as a quality measure.

Dr Welke and colleagues' study shows that volume alone is a poor predictor of outcome. However, after adjustment for patient factors and case complexity, they did identify a complex relationship between surgical volume and outcomes.

There is no relationship between volume and outcome for lowcomplexity cases. However, for complex cases, particularly the Norwood procedure, there was a significant relationship between increasing surgical volume, particularly in very large centers of greater than 350 patients a year, and improved outcomes.

This study complements and supports a previous study by Dr Welke and his colleagues, which was presented at the STS meeting earlier this year. In that study they used an administrative data set and showed that surgeons in centers with a large annual case volume performed more complex cases with better results than those in smaller centers.

Use of case volume as a quality metric is obviously controversial. Previous studies with administrative databases have been criticized because of the lack of data quality and the lack of adequate risk stratification.

The current study Dr Welke and his colleagues used the best available clinical database and the best available risk stratification to identify this relationship between volumes and clinical outcomes.

The goal of the STS database is quality improvement. We have now identified a relationship that some centers might not want to see; that is, there appears to be a relationship, at least for complex cases, between increasing case volume and outcomes. And we have done it using the best available tools.

Now, how can we use this information-you touched on this at the end-for quality improvement? One idea that some payers and other groups might suggest is to simply transfer complex cases from low-volume centers to higher-volume centers. More importantly, we should try to improve quality and improve our outcomes. As you suggested, volume is probably a surrogate for some other factor: surgical experience, available facilities, number of surgeons, or the health care team.

The question I would like to ask is this: How can we use these data to try to identify those factors that we are measuring with volume? Volume is clearly not the determinant but rather is a surrogate for some other outcome measure. How can we identify those factors that account for the improved outcomes in the larger centers and then apply them to other centers to improve overall quality?

Dr Welke. Thank you, Dr Gaynor, for your comments and question.

Volume is not a measure of quality but is, as you stated, an easily obtained structural attribute that is associated with quality. It is a surrogate for process measures and structural characteristics of systems that lead to better outcomes but are not currently captured in available databases. Volume is associated with quality because, on average, higher-volume programs are more likely to have these structural characteristics and engage in these processes. These factors might be associated with experience and systems of care. You mentioned some likely candidates. To those I would add preoperative decision making and care, intraoperative decision making and care, postoperative in-hospital and postdischarge care, multidisciplinary discussions, and team strength and 
experience, including more than 1 surgeon, senior surgeon leadership, skilled cardiologists, pediatric perfusionists, and pediatric anesthesiologists. There are others. I am sure that team experience and interaction are crucial.

Although studying these factors is more time consuming and costly than tracking mortality, defining and implementing such measures are likely to result in better outcomes. How can we do that?

One model that has been very successful in adult cardiac surgery is the regional quality improvement organization, the Northern New England Cardiovascular Disease Study Group. In part because of the geographic dispersion of the programs in our specialty, perhaps a national or international organization such as this one, the STS, or perhaps, most appropriately, the Congenital Heart Surgeons Society, would be best suited to expand on the Northern New England Cardiovascular Disease Study Group concept in our field. It could be a wonderful contribution and might be the only way to tease out the structural characteristics and process measures that are central to high-quality care.

Mortality is a difficult outcome to use for tracking quality, in part because we are good enough at what we do that it occurs relatively infrequently. We need to broaden our focus to include other outcomes, both because they occur more frequently and also because they are important to patients: morbidity, functional status, neurologic status, and long-term mortality.

Dr Jan M. Quaegebeur (New York, NY). Dr Welke, you just put water in my well. Thank you.

I have been involved with the database of New York State, and I want to correct a misconception. The New York State database is not an administrative database because for every patient who undergoes an operation, at the time of the operation, forms have to be filled in and risk factors have to be identified, which are then controlled by the state by means of peer review. Therefore these publications by Hannan that you mentioned already preceded your conclusions, and we totally agree.

I also agree with your last sentence, stating that death, of course, is the ultimate outcome. Therefore the more complex the procedure, the more evident that the relationship exists. If you want to become more sensitive about what really is the quality of care of patients in small and larger units, we have to look at other outcomes than that alone in terms of morbidity, as you mentioned. I think that is very important, but it is not going to be easy to organize that.

Dr Welke. Thank you for your comments, and thank you for your comment about the New York data. Ed Hannan has been one of the pioneers in looking at this subject, as have all of you who have supported him in New York. The New York clinical cardiac surgery database is the only other clinical database that has been used to examine the volume-mortality relationship in pediatric cardiac surgery. The other studies have been done with primarily California administrative data. One study included California and Massachusetts administrative data, and our previous study used data from the Nationwide Inpatient Sample. But as you mentioned, Ed Hannan is a real pioneer and has done great work in this area.

Dr Carlos J. Troconis (Venezuela and Dominican Republic). In the Dominican Republic we have had the experience of and developed a brand new program. In 2006, we did a study in which we evaluated 111 cases performed by our locally trained group versus 169 patients operated on by American teams visiting us. The patients' risk stratifications were the same for both groups: RACHS-1 score up to 3 and Aristotle score of less than 3. In reviewing our results in terms of mortality, we found it within the range applied for most American centers but less than the visitor's teams. In summary, the mortality and morbidity were higher than our result for the same adjusted risk categorization of patients.

My concerns are-and that might be your conclusion - that the different environmental conditions might influence the performance of the visiting teams versus the creativity of "tropicalization" of the local teams habituated to such situations. I think that more collaborative efforts should be done between both worlds to enhance these results. Do you have any comments about this?

Dr Welke. First, I congratulate you on your results. You have brought up an important point. Cardiac surgery is a team sport, and the context matters. If you take one element of a complex team, in this case a group of surgeons from the United States, perhaps even with supporting staff, out of the environment to which they are accustomed and move them to a new environment, in this case your hospital, they might not be able to function optimally. Your environment might be more or less sophisticated than what they are used to at home, but it does not matter. It is just different, and they are not used to it. You and your team are used to the environment, and therefore it makes sense that you get better results.

The answer to improving their results is communication and collaboration. Work with them to adjust them to your environment. Combine their expertise with your experience and expertise. Again, you have brought up an excellent point. What you describe is a case study of what we are discussing: how the team and the whole system matter. 